\title{
Spontaneous Bilateral Intracerebral Hemorrhage Occurring Simultaneously
}

\author{
- Case Report- \\ Masaharu SATO, Shigeru TANAKA, Akitsugu KOHAMA, \\ Teruki SONE*, Masao FUKUNAGA* and Rikushi MORITA* \\ Departments of Emergency Medicine and ${ }^{*}$ Nuclear Medicine, \\ Kawasaki Medical School, Kurashiki, Okayama
}

\begin{abstract}
A 45-year-old male suffered from bilateral putaminal hemorrhages that occurred simultaneously, followed by pseudo-bulbar palsy. Administration of anticoagulants appeared to have caused the bleeding. Cerebral blood flow was markedly decreased in both hemispheres disproportionally to the small size of the hemorrhages, probably due to a bilateral "diaschisis phenomenon," and the patient experienced severe neurological sequelae.
\end{abstract}

Key words: cerebral blood flow, single photon emission computed tomography, spontaneous intracerebral hemorrhage

\section{Introduction}

Recurrence of spontaneous intracerebral hemorrhage is sometimes encountered. However, multiple simultaneous hemorrhages are very rare; to date, only two cases have been reported. ${ }^{5,6)}$ We treated a patient in whom spontaneous bilateral intracerebral hemorrhages occurred simultaneously.

\section{Case Report}

On February 1, 1984, a 45-year-old male suddenly experienced weakness of the right upper limb and motor dysphasia. He was admitted to a local hospital, where a computed tomographic (CT) scan revealed no abnormal density. His symptoms disappeared within 3 weeks and he was discharged on March 1, 1984. On March 7, 1984, a follow-up CT scan revealed a low-density spot in the left putamen. Therefore, the previous symptoms were thought to be a reversible ischemic neurological deficits caused by a lacunar stroke. Then, the patient was given anticoagulants intravenously three times a week. The prescribing physician was unable to inform us which anticoagulants were administered.

Received July 25, 1985; Accepted March 17, 1986
Although, gingival and nasal bleeding occurred frequently, the anticoagulant therapy was continued until August 10, 1984, when the patient, while taking a bath, suddenly developed left hemiparesis and speech disturbance. He was referred to our clinic and admitted.

On admission he was alert and well oriented but he exhibited left hemiplegia, which also affected his face and tongue, left hemihypesthesia, dysphagia, motor aphasia, and dysarthria. The history contained no evidence of predisposing factors; he had neither hypertension nor diabetes mellitus. A CT scan revealed bilateral putaminal hemorrhages, which were larger on the right side (Fig. 1A). The left hemorrhage occurred in the putaminal lacuna. Laboratory data showed no abnormalities. The gingival and nasal bleeding had stopped. He became able to walk with a cane in one month under conservative therapy, and was transferred to the Department of Rehabilitation for further physical therapy.

On October 9, 1984, a CT scan showed bilateral, localized low-density areas in the basal ganglia (Fig. 1B). Regional cerebral blood flow was assessed by single photon emission $\mathrm{CT}$ with intravenous administration of $\mathrm{N}$-isopropyl-[ $\left.{ }^{123} \mathrm{I}\right]$-p-iodoamphetamine, ${ }^{21}$ and the image revealed a marked decrease in regional cerebral blood flow over a wide area of the 

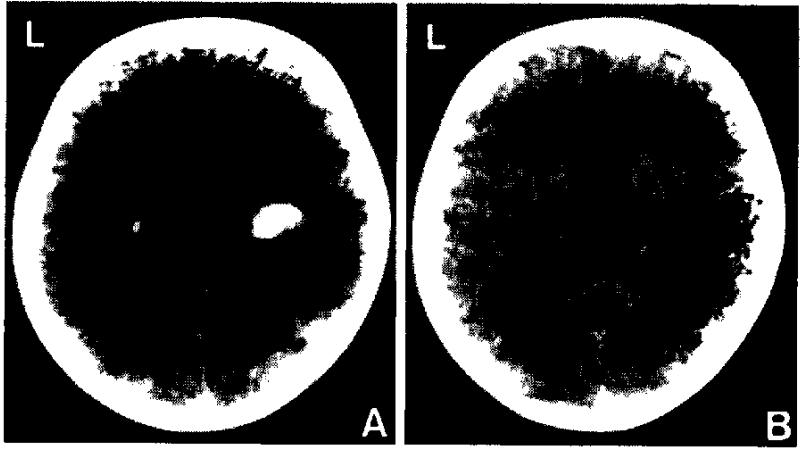

Fig. 1 A: A CT scan on admission showed bilateral putaminal hemorrhages. B: A CT scan 2 months after onset revealed low-density areas in the bilateral basal ganglia.

bilateral basal ganglia (Fig. 2). As of this writing, the patient can walk with a cane. However, motor dysphasia, dysphagia, dysarthria, and left hemiparesis, mainly in the upper limb, persist.

\section{Discussion}

Simultaneous multiple bleeds within the central nervous system are very rare. To date only two cases have been reported ${ }^{5,6)}$ (Table 1). The mechanisms of simultaneous hemorrhage seem to vary. In Case 1, the simultaneous bleeds occurred just after the evacuation of a left chronic subdural hematoma; rapid decompression of high intracranial pressure might play an important role in marked bilateral hyperperfusion, resulting in bilateral hemorrhage. The cause was not found in Case $2 .{ }^{6)}$ In our patient, the administration of anticoagulants apparently triggered the bilateral hemorrhages.

When bleeding occurs in one hemisphere, cerebral blood flow in the contralateral hemisphere may be

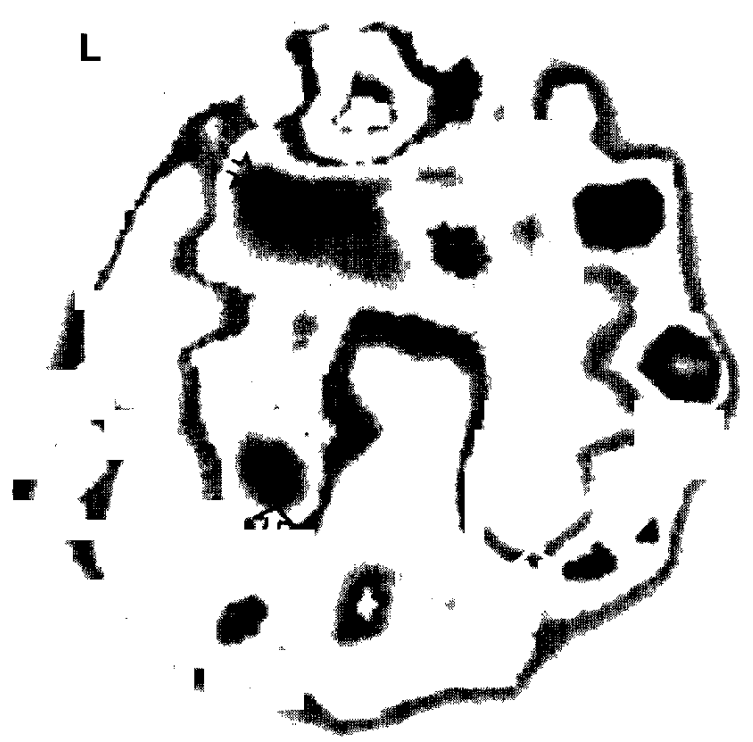

Fig. 2 Regional cerebral blood flow was assessed by single photon emission $\mathrm{CT}$ using $\mathrm{N}$-isopropyl$\left[{ }^{123} \mathrm{I}\right]$-p-iodoamphetamine. There was a marked bilateral decrease over a wide area in the basal ganglia (arrows).

decreased due to the "diaschisis phenomenon." The neurological deficit, e.g., hemiparesis, will gradually, to some degree, abate because the noncrossing pyramidal tract and the sensory factor may compensate for the neurological deficit. ${ }^{1,3)}$ If an additional bleed occurs in the contralateral hemisphere, several phenomena will develop. First, the symptom that existed before the recurrence of bleeding will worsen, owing to breakdown of the sensory factor, which had played an important part in compensating for the original symptom. ${ }^{1,3)}$ Second, the non-cross-

Table 1 Simultaneous bilateral intracerebral hemorrhage-clinical data

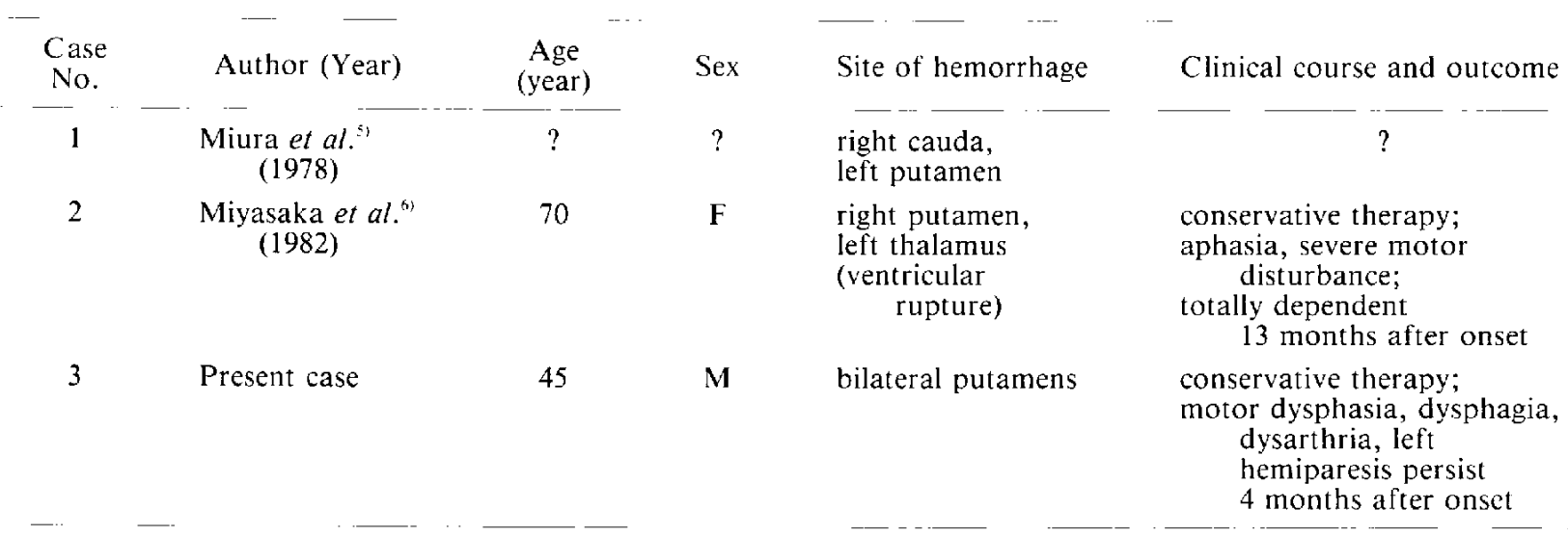


ing pyramidal tract may be disturbed by the recurrent bleeding. Third, cerebral blood flow may be reduced in both hemispheres because the "diaschisis phenomenon" may be brought about bilaterally. This, in turn, will further compromise the residual function of the sensory factor and the non-crossing pyramidal tract. Simultaneous bilateral hemorrhages cause these phenomena at the same time, resulting in severe hypofunction of both hemispheres, as shown in our case.

In our patient, measurement of regional cerebral blood flow indicated that the disturbance in distribution of $\mathrm{N}$-isopropyl-[ $\left.{ }^{123} \mathrm{I}\right]$-p-iodoamphetamine covered a much wider area in both hemispheres than was anticipated from the size of the hematomas, which did not demonstrate a mass effect on routine CT scanning. Thus, a bilateral "diaschisis phenomenon" was indicated by the cerebral blood flow measurement, and this probably accounted for the severe sequelae.

\section{Acknowledgment}

The authors wish to thank Nihon Mediphysics for supplying $\mathrm{N}$-isopropyl-[ $\left.{ }^{123} \mathrm{I}\right]$-p-iodoamphetamine.

\section{References}

1) Fisher CM, Curry HB: Pure motor hemiplegia of vascular origin. Arch Neurol (Chicago) 13: 30-44, 1965

2) Kuhl DE, Barrio JR, Huang SC, Selin C, Ackermann RF, Lear JL, Wu JL, Lin TH, Phelps ME: Quantifying local cerebral blood flow by $\mathrm{N}$-isopropyl-p-[1-123]iodoamphetamine (IMP) tomography. $J$ Nucl Med 23: 196-203, 1982

3) Lassek AM, Emery SL: Hidden neurologic mechanisms in motor function restitution. Neurology (Minneap) 9: 107-110, 1959

4) Meyer JS, Shinohara $Y$, Kanda $T$, Fukuuchi $Y$, Ericsson AD, Kok NK: Diaschisis resulting from acute unilateral cerebral infarction. Arch Neurol 23: 241247, 1970

5) Miura N, Nakahara A, Kagawa M, Kitamura K, Kobayashi N: A study of hypertensive intracerebral hemorrhage. No Shinkei Geka 6: 635-645, 1978 (in Japanese)

6) Miyasaka Y, Nakayama K, Matsumori K, Beppu T, Kurata A, Satoh K, Kitahara T, Morii S, Owada T, Yada K: Bilateral hypertensive intracerebral hemorrhage diagnosed by CT scan. Report of five cases and review of literature. Neurol Med Chir (Tokyo) 22: $661-667,1982$ (in Japanese)

Address reprint requests to: M. Sato, M.D., Department of Emergency Medicine, Kawasaki Medical School, 577 Matsushima, Kurashiki, Okayama 701-01, Japan. 